\title{
Thermal and Mechanical Properties of New Main-Chain Liquid-Crystalline Elastomers
}

\author{
Antoni Sánchez-Ferrer ${ }^{1,2}$ and Heino Finkelmann ${ }^{2}$ \\ ${ }^{1}$ Physics Department, University of Fribourg, Fribourg, Switzerland \\ ${ }^{2}$ Institute for Macromolecular Chemistry, Albert Ludwigs University, \\ Freiburg, Germany
}

New Main-Chain Liquid-Crystalline Elastomers (MCLCEs) were synthesised based on reacting vinyloxy-terminated mesogens under hydrosilylation conditions with a flexible crosslinker. These main-chain systems showed smectic and nematic mesophases and their anisotropic properties were mechanically and thermally analysed as function of the crosslinking density. Due to the suitable chemistry used in this work low crosslinking densities have been achieved (2.5 mol-\%) with low soluble content $(5 \%)$. For the first time, the degree of crosslinking could be adjusted and nematic or smectic MCLCEs with tuneable thermal and mechanical properties were obtained.

Keywords: liquid-crystalline elastomers; thermoelastic experiments; uniaxial stressstrain deformations

\section{INTRODUCTION}

In the early '80s, Aguilera and Ringsdorf [1] succeeded in synthesising Main-Chain Liquid-Crystalline Polymers (MCLCPs) based on three aromatic rings mesogenic units and oligosiloxanes as chain extenders. This kind of chemistry reduced the glass transition $\left(T_{g}\right)$ and clearing temperatures $\left(T_{c}\right)$ of those MCLCPs due to the flexibility of the siloxane spacer. Years later, Donnio, Wermter, and Finkelmann [2] obtained the first Main-Chain Liquid-Crystalline Elastomers (MCLCEs) by crosslinking the liquid-crystalline polymer chains with a flexible siloxane-based crosslinker. In both studies, low degrees of

Address correspondence to Antoni Sánchez-Ferrer, Physics Department, University of Fribourg, Chemin du Musée, 3, 1700 Fribourg, Switzerland. E-mail: antonio. sanchez-ferrer@unifr.ch 
polymerisation were obtained, from 6 to 9 repeating units, as it could be noticed from the molecular weights of the polymers and from the high percentages of soluble content and crosslinker used in the networks.

MCLCEs are of interest due to the coupling of the polymer backbone to the director [3-4]; the monodomains of these systems have huge thermal expansion, up to $400 \%$, with respect to the Side-Chain Liquid-Crystalline Elastomers (SCLCEs). This impressive property is of high interest for the scientific community because of the potential uses as actuators [5-6] or artificial muscles [7-9]. In the process, external stimuli are needed, like thermal gradients, magnetic or electrostatic fields, or light. Several approaches have been taken for the integration of conductive particles in order to increase their conductivity [10] or photo-isomerisable dies that destroy the liquid-crystalline phase upon change their shape from trans- to cis-isomer [11-12].

Main-chain elastomers are much more difficult to fabricate and work with than side-chain elastomers. A key point is the soluble content of the material during the polycondensation reaction, possibly determined by side reactions in the polymerisation and impurities that did not allow obtaining well-defined networks [2-4,13] and directly affected the mechanical and thermal properties of these materials.

We present the synthesis of well-defined monodomain samples of MCLCEs obtained by hydrosilylation of new mesogens. In order to synthesize networks, the solution was found in the use of vinyloxy carbon chains. The use of vinyloxy alkyl chains allowed the formation of networks where the soluble content was minimised with respect to the common vinyl ones. The mechanical properties and the thermal actuation of these elastomers are presented and studied in detail in order to understand better these fantastic materials.

\section{RESULTS AND DISCUSSION}

\subsection{Synthesis of MCLCEs}

In the synthesis of all MCLCEs, a flexible crosslinker with 5 reacting groups was used for preparing samples with 10 and $2.5 \mathrm{~mol}-\%$ of crosslinker. Then, for samples with 10 and $2.5 \mathrm{~mol}-\%$, the number of mesogens on average in the polymer chains $(D P)$ would be 4 and 16 respectively. From the experience in the synthesis of MCLCPs, the degree of polymerisation for the linear polymers is higher than for the networks. Thus, well-defined elastomers were obtainable using 
these crosslinker quantities, even taking possible defects on building the network into consideration.

The procedure in the synthesis of MCLCEs was the same as for SCLCEs, but with longer reaction times. Mixtures of mesogen, chain-extender (TMDSO), crosslinker (PMPOPS) and azobenzene co-monomer (Azo) - for photoactive elastomers - were prepared and partially crosslinked. The swollen elastomer strip was removed from the spin-casting cell and aligned by applying a strong uniaxial stress and then elongated to uniformly align with the director. Then, the unswollen sample was completely cured to fix the monodomain orientation in the case of nematic elastomers and the conical distribution of domains in the case of smectic-C elastomers. In Scheme 1, the molecules involved in the synthesis of the four MCLCEs are shown.

The final compositions of the four elastomers were the following: SmC-MCE-10 ( $\mathrm{a}=10 \mathrm{~mol}-\%$; $\mathrm{c}=100 \mathrm{~mol}-\%)$, SmC-MCE-2.5 $(\mathrm{a}=$ $2.5 \mathrm{~mol}-\% ; \quad b=5 \mathrm{~mol}-\% ; \quad c=95 \mathrm{~mol}-\%), \quad \mathrm{N}-\mathrm{MCE}-10 \quad(\mathrm{a}=10 \mathrm{~mol}-\mathrm{-} \%$; $\mathrm{b}=5 \mathrm{~mol}-\%$; $\mathrm{c}=95 \mathrm{~mol}-\%)$, and N-MCE- $2.5(\mathrm{a}=2.5 \mathrm{~mol}-\%$; $\mathrm{b}=5 \mathrm{~mol}-\%$; $\mathrm{d}=95 \mathrm{~mol}-\mathrm{-} \%$ ); where all the values are the mol- $\%$ related to the total amount of mesogenic units.

\subsection{Thermal Properties of MCLCEs}

MCLCEs have larger uniaxial thermal responses (up to $400 \%$ ) than side-chain systems (Fig. 1). Thermoelastic experiments were done for correlating the crosslinking density of the samples with the uniaxial thermal expansion $\left(L / L_{\mathrm{ISO}}\right)$ and the soluble content. Prolate monodomain samples of MCLCEs with vinyloxy chains showed both mesophases: smectic and nematic (Fig. 2), depending on the chemistry of the mesogen. On decreasing the quantity of crosslinker, the clearing temperature of the smectic elastomers decreased, while the opposite took place for the nematic elastomers (Table 1).

The uniaxial thermal expansion was indirectly proportional to the crosslinking density: more crossliker, less expansion. Nematic samples with the same percentage of crosslinker expanded less than the corresponding smectics due to their lower order $(S=0.73)$ as compared to the smectic layering order $(S=0.83)$ where the polymer chains are more stretched.

\subsection{Mechanical Properties of MCLCEs}

To answer the very important questions whether MCLCEs differ from SCLCEs in their mechanical properties, and how the crosslinking 
<smiles>C[SiH]1O[SiH2]O[SiH](C)O[SiH](C)O[SiH](C)O1</smiles>

a) PMPOPS

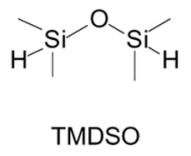

TMDSO<smiles>C=CCCCCOc1ccc(/N=N/c2ccc(OCCCCC=C)cc2)cc1</smiles>

b) Azo<smiles>[R]Oc1ccc(OC(=O)c2ccc(O[R]C=C)cc2)cc1</smiles>

c) main-chain mesogen 1

$$
\mathrm{R}=\left(\mathrm{CH}_{2}\right)_{4} \mathrm{O}
$$<smiles>[R]C=COc1ccc(C(=O)Oc2ccc(OC(=O)c3ccc(OC=C)cc3)cc2CC)cc1</smiles>

d) main-chain mesogen 2

$$
\mathrm{R}=\left(\mathrm{CH}_{2}\right)_{4} \mathrm{O}
$$

SCHEME 1 Chemical structures of the flexible crosslinker, chain extender, azobenzene dye, and main-chain monomers for the obtaining of MCLCEs.

density influences the liquid-crystalline phase, stress-strain experiments on nematic and smectic MCLCEs were performed on samples with different crosslinking densities as basis for the correlation of opto-mechanical and opto-thermoelastic experiments.

In a recent communication [14], the stress-strain non-linear behaviour of nematic MCLCEs using benzophenone moieties as crosslinkers was reported. A single exponential function with a linear correction term was assumed. For our samples, an exponential growth is introduced in order to analyse the pre-stress region in smectic elastomers and give a general function for both kind of samples, nematic or 


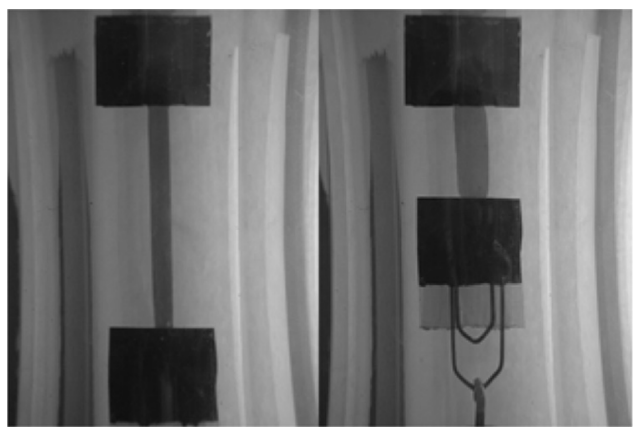

FIGURE 1 Change in length of a main-chain liquid-crystalline elastomer: From the liquid-crystalline phase to the isotropic phase.

smectic. Thus, the equation that describes the increase of true stress as function of the applied strain is given by

$$
\sigma(\lambda)=\sigma_{\text {lin }}+\sigma_{\text {exp }}+\sigma_{\text {pre }}=\mathrm{a}(\lambda-1)+\mathrm{b}\left(e^{\mathrm{c}(\lambda-1)}-1\right)+\mathrm{d}\left(1-e^{-\mathrm{f}(\lambda-1)}\right)
$$

The fitting parameter "a" corresponds to the linear behaviour of Young's modulus, "b" and "c" are the fitting parameters of the exponential growth, and " $d$ " and "f" are the fitting parameters of the pre-stress region that can be applied for smectic elastomers.

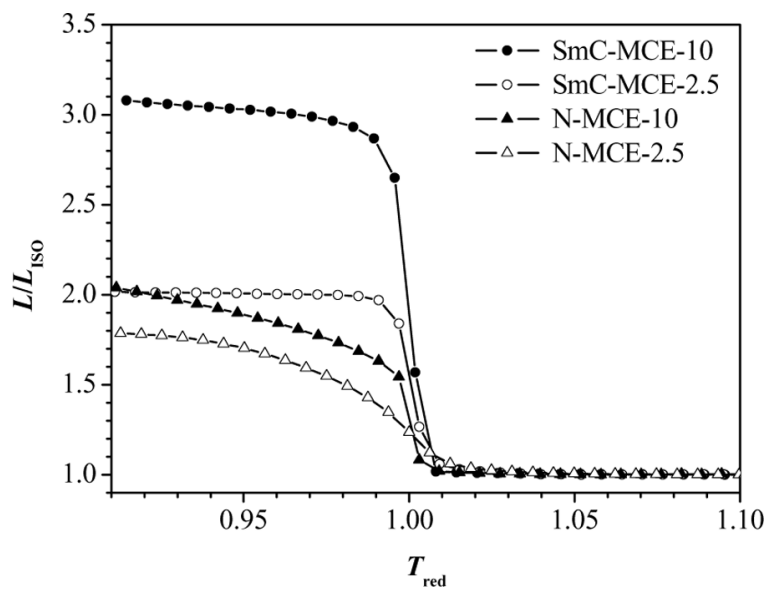

FIGURE 2 Uniaxial thermal expansion $\left(L / L_{\mathrm{ISO}}\right)$ as function of reduced temperature $\left(T_{\text {red }}=T / T_{\mathrm{c}}\right)$ for the four MCLCEs. 
TABLE 1 Clearing Temperature $\left(T_{\mathrm{c}}\right)$, Thermal Expansion Differences $\left(L / L_{\text {ISO }}\right)$ at the Reduced Temperature $T_{\text {red }}=0.92$, Order Parameter $(S)$, and Soluble Content $(s c)$ of the Four Elastomers

\begin{tabular}{lcccc}
\hline Samples & $T_{\mathrm{C}}\left({ }^{\circ} \mathrm{C}\right)$ & $L / L_{\text {ISO }}(0.92)$ & $S$ & $s c(\%)$ \\
\hline SmC-MCE-10 & $53(\mathrm{SI})$ & 2.02 & 0.83 & 5.0 \\
SmC-MCE-2.5 & 47 (SI) & 3.07 & 0.78 & 5.7 \\
N-MCE-10 & $48(\mathrm{NI})$ & 1.78 & 0.73 & 3.8 \\
N-MCE-2.5 & $55(\mathrm{NI})$ & 2.02 & 0.73 & 4.9 \\
\hline
\end{tabular}

The Young's modulus is calculated from the derivative of Eq. (1) giving the following equation:

$$
E(\lambda)=\frac{\partial \sigma_{\mathrm{t}}}{\partial \lambda}=E_{\text {lin }}+E_{\text {exp }}+E_{\text {pre }}=\mathrm{a}+\mathrm{b} \cdot \mathrm{c} \cdot e^{\mathrm{c}(\lambda-1)}+\mathrm{d} \cdot \mathrm{f} \cdot e^{-\mathrm{f}(\lambda-1)}
$$

Nematic MCLCEs (N-MCE-10 and N-MCE-2.5) showed higher mechanical responses than side-chain systems. For example, SCLCEs had polymer chain lengths of $30 \AA$ (8 repeating units) for $10 \mathrm{~mol}-\%$ of crosslinker. Their corresponding Young's moduli were in the range of $0.6 \mathrm{MPa}$ at $T_{\text {red }}=0.92$ in these systems. MCLCEs had polymer chain lengths of $137 \AA$ (4 repeating units) for $10 \mathrm{~mol}-\%$ of crosslinker (N-MCE-10) and $632 \AA$ (16 repeating units) for 2.5 mol- $\%$ of crosslinking density (N-MCE-2.5). Their corresponding initial Young's moduli were $E_{0}=2.6 \mathrm{MPa}$ and $0.3 \mathrm{MPa}$, respectively, at $T_{\text {red }}=0.92$ (Fig. 3). Thus, MCLCEs had a clear different mechanical behaviour due to their chemical polymer chain constitution with respect to SCLCEs.

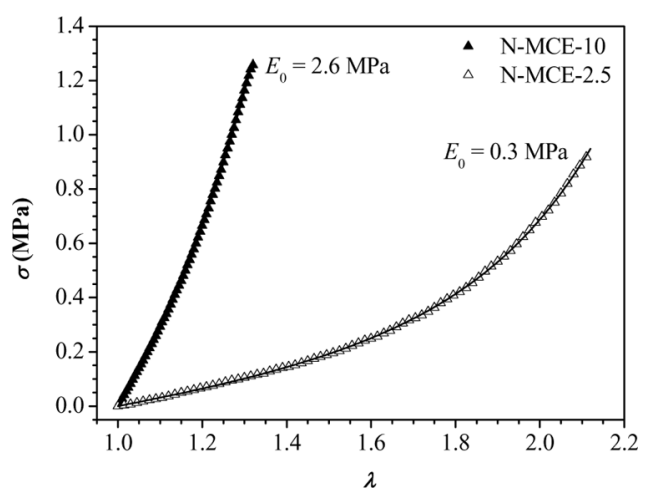

FIGURE 3 Uniaxial stress-strain curves for the samples N-MCE-10 and N-MCE-2.5 at $T_{\text {red }}=0.92$ (nematic phase). 
Smectic elastomers presented a difference in their mechanical properties with respect to the nematics, where all them had a pre-stress transformation region. These samples $-10 \mathrm{~mol}-\%$ (SmC-MCE-10) and $2.5 \mathrm{~mol}-\%$ (SmC-MCE-2.5) of crosslinker - showed initial Young's moduli of $E_{0}=11.2 \mathrm{MPa}$ and $17.4 \mathrm{MPa}$, respectively, at $T_{\text {red }}=0.92$ (Fig. 4), and only the samples with $10 \mathrm{~mol}-\%$ of crosslinker presented a linear behaviour.

The explanation given by Krause and Finkelmann [14] can be also applied in this case. The main difference with respect to their system is that the crosslinking process takes place at the same time as the polymerisation. Thus, the polymer chain distribution is narrower than the distribution of chains between the crosslinking points under photo-irradiation conditions. The final result is also a hardening of the sample: less than the UV-photo-crosslinked systems, but more that the hairpins model by Adams and Warner [15].

Smectic samples presented almost the same behaviour but with and extra factor: the pre-stress transformation region. The fact that smectic samples showed this behaviour can be explained by an extra energetic factor due to the smectic layering.

In nematic elastomers, the Young's modulus is proportional to the degree of crosslinking due to the entropy elasticity already described by Warner, Terentjev and Lubensky [16-18]. For smectic samples, the elasticity of the systems is inversely proportional to their crosslinking density, where the enthalpy elasticity factor from the smectic layers plays an important role [19-21]. This can be explained by the

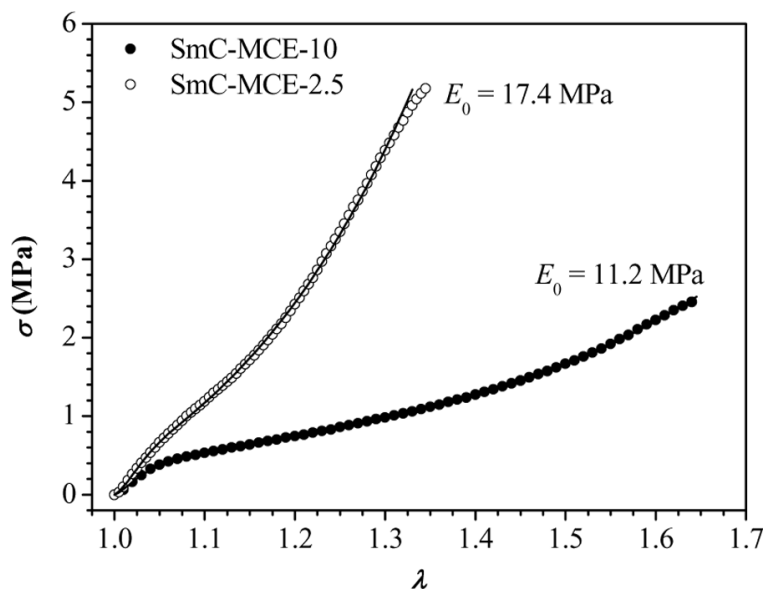

FIGURE 4 Uniaxial stress-strain curves for the samples SmC-MCE-10 and SmC-MCE-2.5 at $T_{\text {red }}=0.92$ (smectic-C phase). 
fact that at high concentration of crosslinker the smectic layers get disturbed because the polymer chains between crosslinking points are smaller and have less mobility for the obtaining of smectic phases: as a consequence the enthalpy diminishes. Thus, the mechanical response of highly crosslinked smectic elastomers was less than that of the low crosslinked ones.

In principle, nematic mesogens would give nematic elastomeric systems and smectic mesogens would result into their corresponding smectic ones. The presence of $5 \mathrm{~mol}-\%$ of azo-compound as a doping molecule was only affecting highly crosslinked smectic elastomers, where one could see this effect on passing from the smectic-C sample SmC-MCE-10 to the cybotactic nematic N-MCE-10. It is known that mixtures of mesogens with different number of carbons in the chains induce disorder in the systems, and nematic phases can be obtained by mixing mesogens that give crystalline or smectic elastomers when their homopolymer networks are synthesized [22]. Thus, 5 mol- $\%$ of the azobenzene derivative did not influence the phase behaviour at low crosslinking density but nematic phase was induced at higher crosslinker concentration.

In a new communication [23], the properties of smectic-C mainchain elastomers under uniaxial and shear deformations are discussed in detail because of the new physics involved in these processes.

\section{CONCLUSIONS}

New Main-Chain Liquid-Crystalline Elastomers (MCLCEs) were synthesized from vinyloxy-terminated monomers using the spincasting technique. This new chemistry avoids side reactions and allows obtaining well-defined networks with low soluble content compared to the previous systems with olefinic mesogens.

Thermoelastic experiments confirmed that all samples studied (MCLCEs) presented a prolate conformation of the backbone polymer chains, which induced a strong anisotropy along the stretched direction parallel to the director. All elastomers expanded on cooling and contracted on heating, because of the direct proportionality between the uniaxial thermal expansion and the order parameter of the sample.

In the case of MCLCEs the thermal expansion differences went from $80 \%$ to $210 \%$. Two types of elastomers were studied: smectic and nematic. Smectic elastomers had a discontinuity in the clearing temperature, while a continuous behaviour of the change in length was observed for nematic elastomers. Another fact noticed was that the presence of flexible crosslinker increases the clearing temperature in main-chain systems. 
Uniaxial stress-strain deformations were done in order to study mechanical properties on these LCEs. An exponential behaviour was observed for nematic elastomers and a pre-stress transformation region was analysed for the smectic ones. Stress-strain experiments on nematic elastomers were correlated with the chemical constitution of the samples, showing a direct proportionally relation between the degree of crosslinking and the elastic modulus, and the exact opposite for the smectic elastomers.

Most importantly, the mechanical properties (elastic modulus) and the spontaneous contraction (uniaxial thermal expansion) can now be tuned/trigged as function of the chemistry of the mesogen and crosslinking density.

\section{REFERENCES}

[1] Aguilera, C. \& Ringsdorf, H. (1984). Polym. Bull., 12, 93.

[2] Donnio, B., Wermter, H., \& Finkelmann, H. (2000). Macromolecules, 33, 7724.

[3] Wermter, H. \& Finkelmann, H. (2001). e-Polymers, 013, 1.

[4] Tajbakhsh, A. R. \& Terentjev, E. M. (2001). Eur. Phys. J. E, 6, 181.

[5] Fischl, T., Albrecht, A., Wurmus, H., Hoffmann, M., Stubenrauch, M., \& Sánchez-Ferrer, A. (2006). Kunststoffe, 96, 30.

[6] Bründel, M., Stubenrauch, M., Wurmus, H., \& Sánchez-Ferrer, A. (2004). International Newsletter on Micro-Nano Integration (MST-NEWS), 4, 38.

[7] de Gennes, P. G., Hebert, M., \& Kant, R. (1997). Macromol. Symp., 113, 39.

[8] Shenoy, D. K., Thomsen III, D. L., Srinivasan, A., Keller, P., \& Ratna, B. R. (2002). Sensors and Actuators A, 96, 184.

[9] Naciri, J., Srinivasan, A., Jeon, H., Nikolov, N., Keller, P., \& Ratna, B. R. (2003). Macromolecules, 36, 8499.

[10] Chambers, M., Zalar, B., Remskar, M., Zumer, S., \& Finkelmann, H. (2006). Appl. Phys. Lett., 89, 243116.

[11] Finkelmann, H., Nishikawa, E., Pereira, G. G., \& Warner, M. (2001). Phys. Rev. Lett., 87, 015501.

[12] Hogan, P. M., Tajbakhsh, A. R., \& Terentjev, E. M. (2002). Phys. Rev. E, 65, 041720.

[13] Rousseau, I. A. \& Mather, P. T. (2003). J. Am. Chem. Soc., 125, 15300.

[14] Krause, S., Dersch, R., Wendorff, J. H., \& Finkelmann, H. (2007). Macromol. Rapid Commun., 28, 2062.

[15] Adams, J. M. \& Warner, M. (2005). Eur. Phys. J. E, 16, 97.

[16] Golubovic, L. \& Lubensky, T. C. (1989). Phys. Rev. Lett., 63, 1082.

[17] Warner, M., Gelling, K. P., \& Vilgis, T. A. (1988). J. Chem. Phys., 88, 4008.

[18] Warner, M. \& Terentjev, E. M. (1996). Progress Polymer Sci., 21, 853.

[19] Nishikawa, E., Finkelmann, H., \& Brand, H. R. (1997). Macromol. Rapid Commun., 18,65 .

[20] Aßfalg, N. \& Finkelmann, H. (2001). Macromol. Chem. Phys., 202, 794.

[21] Warner, M. \& Terentjev, E. M. (2007). Liquid Crystal Elastomers, Oxford University Press: USA, 310.

[22] Brandt, H. (2004). Static- and Dynamic-Mechanical Analysis in New Main-Chain Liquid-Crystalline Networks, University of Freiburg: Albert Ludwigs.

[23] Sánchez-Ferrer, A. \& Finkelmann, H. (2008). Macromolecules, 41, 970. 